\title{
A REMARK ON WEIGHTED INEQUALITIES FOR GENERAL MAXIMAL OPERATORS
}

\author{
C. PÉREZ
}

(Communicated by J. Marshall Ash)

\begin{abstract}
Let $1<p<\infty$, and let $w, v$ be two nonnegative functions. We give a sufficient condition on $w, v$ for which the general maximal operator $M_{\mathscr{B}}$ is bounded from $L^{p}(v)$ into $L^{p}(w)$. Our condition is stronger but closely related to the $A_{p, \mathscr{B}}$ condition for two weights.
\end{abstract}

\section{INTRODUCTION}

Let $\mathscr{Q}$ be the family of all open cubes in $\mathbf{R}^{n}$ with sides parallel to the axes, and let $M_{\mathscr{Q}}=M$ denote the Hardy-Littlewood maximal operator. According to a fundamental theorem of Sawyer [11], $M$ is a bounded operator from $L^{p}(v)$ into $L^{p}(w), 1<p<\infty$, if and only if $(w, v) \in S_{p}$, that is, there is a positive constant $c$ such that

$$
\int_{Q} M\left(v^{1-p^{\prime}} \chi_{Q}\right)(y)^{p} w(y) d y \leq c \int_{Q} v(y)^{1-p^{\prime}} d y, \quad Q \in \mathscr{Q} .
$$

On the other hand, it is well known that Muckenhoupt's $A_{p}$ condition for two weights,

$$
\left(\frac{1}{|Q|} \int_{Q} w(y) d y\right)^{1 / p}\left(\frac{1}{|Q|} \int_{Q} v(y)^{1-p^{\prime}} d y\right)^{1 / p^{\prime}} \leq c, \quad Q \in \mathscr{Q},
$$

is not equivalent to $S_{p}$ unless $v=w$ (cf. [3; 2, p. 433]). One problem with Sawyer's condition is that it is very difficult to test in practice since it involves the operator $M$ on it. It would be interesting to obtain sufficient conditions close in form to (2). In [10] we initiated this program and showed that it is enough to consider conditions such as (2) but replacing the local $L^{p^{\prime}}$ average norm involved on the weight $v$ by appropriate stronger norms. An antecedent of these results can be found in Neugebauer's paper [8].

This note is devoted to studying the corresponding two weight problem

$$
\int_{\mathbf{R}^{n}} M_{\mathscr{B}} f(y)^{p} w(y) d y \leq c \int_{\mathbf{R}^{n}}|f(y)|^{p} v(y) d y,
$$

where $\mathscr{B}$ is a general basis. By a basis $\mathscr{B}$ in $\mathbf{R}^{n}$, we mean a collection of open sets in $\mathbf{R}^{n}$. The study of general maximal operators $M_{\mathscr{B}}$ arises in many

Received by the editors April 18, 1991 and, in revised form, May 28, 1991.

1991 Mathematics Subject Classification. Primary 42B25. 
situations in Fourier Analysis where the geometry involved is other than the one given by the cubes or balls. Of course, in one-parameter Fourier Analysis the basis $\mathscr{Q}$ plays a central role together with the basis $\mathscr{D}$ of all dyadic cubes. A corresponding role in multiparameter Fourier Analysis is played by the basis $\mathscr{R}$ of all rectangles with sides parallel to the axes. An example of an "exotic" but interesting basis is given by the Córdoba-Zygmund basis $\Re$ in $\mathbf{R}^{3}$ of all rectangles with sidelengths of the form $\{s, t, s t\}$.

For a general basis $\mathscr{B}, M_{\mathscr{B}}$ denotes the maximal operator associated to $\mathscr{B}$ defined by

$$
M_{\mathscr{B}} f(x)=\sup _{x \in B} \frac{1}{|B|} \int_{B}|f(y)| d y
$$

if $x \in \bigcup_{B \in \mathscr{B}}$ and $M_{\mathscr{B}} f(x)=0$, otherwise. We say that $w$ is a weight associated to the basis $\mathscr{B}$ if $w$ is a nonnegative measurable function in $\mathbf{R}^{n}$ such that $w(B)=\int_{B} w(y) d y<\infty$ for each $B$ in $\mathscr{B}$. The weight $w$ belongs to the class $A_{p, \mathscr{B}}, 1<p<\infty$, if there is a constant $c$ such that

$$
\left(\frac{1}{|B|} \int_{B} w(y) d y\right)\left(\frac{1}{|B|} \int_{B} w(y)^{1-p^{\prime}} d y\right)^{p-1} \leq c
$$

for all $B \in \mathscr{B}$. For $p=\infty$, we set $A_{\infty, \mathscr{B}}=\bigcup_{p>1} A_{p, \mathscr{B}}$.

In [9] we introduced the following class of bases that we shall use later.

Definition 1.1. A basis $\mathscr{B}$ is a Muckenhoupt basis if for each $1<p<\infty$, and every $w \in A_{p, \mathscr{B}}$,

$$
M_{\mathscr{B}}: L^{p}(w) \rightarrow L^{p}(w)
$$

It is shown in [9] that this definition is equivalent to saying that for each $1<p<\infty$

$$
M_{\mathscr{B}, w}: L^{p}(w) \rightarrow L^{p}(w), \quad w \in A_{\infty, \mathscr{B}} .
$$

Here $M_{\mathscr{B}, w}$ denotes the weighted maximal operator defined by

$$
M_{\mathscr{B}, w} f(x)=\sup _{x \in B} \frac{1}{w(B)} \int_{B}|f(y)| w(y) d y .
$$

Most of the important bases are Muckenhoupt bases, and, in particular, those mentioned above: $\mathscr{Q}, \mathscr{D}, \mathscr{R}$, and $\mathfrak{R}$.

Now let $(w, v)$ be a couple of weights associated to the basis $\mathscr{B}$. Extending a previous result of Sawyer in [12] for the strong maximal operator, Jawerth [4] gave a necessary and sufficient condition for $M_{\mathscr{B}}$ to be bounded from $L^{p}(v)$ into $L^{p}(w)$ under no restriction on $\mathscr{B}$. Let $\mathscr{F}$ be the family of all finite unions $G=\bigcup_{j=1}^{N} B_{j}$ of sets in $\mathscr{B}$; Jawerth's condition is that for some positive constant $c$

$$
\int_{G} M_{\mathscr{B}}\left(v^{1-p^{\prime}} \chi_{G}\right)(y)^{p} w(y) d y \leq c \int_{G} v(y)^{1-p^{\prime}} d y, \quad G \in \mathscr{F} .
$$

This condition is even harder to verify than Sawyer's condition $S_{p}$. In Theorem 1.3 we shall provide a simpler sufficient condition that is not necessary.

We shall use the following class of weights. 
Definition 1.2. We say that a weight $w$ associated to the basis $\mathscr{B}$ satisfies condition (A) if there are constants $0<\lambda<1,0<c=c(\lambda)<\infty$ such that for all measurable sets $E$

$$
w\left(\left\{x \in \mathbf{R}^{n}: M_{\mathscr{B}}\left(\chi_{E}\right)(x)>\lambda\right\}\right) \leq c w(E) .
$$

Before stating our main theorem we shall make some remarks concerning condition (A).

This class of weights was considered by Jawerth in [4], although the unweighted version goes back to the work of Córdoba in [1]. One reason that makes condition (A) interesting is the fact that it is weaker than the $A_{\infty, \mathscr{B}}$ condition whenever the basis $\mathscr{B}$ is a Muckenhoupt basis. To see this let $w \in A_{\infty, \mathscr{B}}$. Then $w \in A_{r, \mathscr{B}}$ for some $1<r<\infty$ and, by standard properties of the $A_{p, \mathscr{B}}$ weights,

$$
\frac{|E|}{|B|} \leq c\left(\frac{w(E)}{w(B)}\right)^{1 / r}
$$

for each measurable subset $E \subset B \in \mathscr{B}$. It follows then that $M_{\mathscr{B}}\left(\chi_{E}\right)(x) \leq$ $c\left(M_{\mathscr{B}, w}\left(\chi_{E}\right)(x)\right)^{1 / r}$, and, therefore, if $\mathscr{B}$ is a Muckenhoupt basis then (5) yields, for all $\lambda>0$,

$$
\begin{aligned}
w\left(\left\{x \in \mathbf{R}^{n}: M_{\mathscr{B}}\left(\chi_{E}\right)(x)>\lambda\right\}\right) & \leq w\left(\left\{x \in \mathbf{R}^{n}: M_{\mathscr{B}, w}\left(\chi_{E}\right)(x)>\frac{\lambda^{r}}{c^{r}}\right\}\right) \\
& \leq \frac{c^{r}}{\lambda^{r}} \int_{\mathbf{R}^{n}} \chi_{E}(x)^{r} w(x) d x=c(\lambda) w(E),
\end{aligned}
$$

which is condition $(\mathrm{A})$.

In fact Jawerth and Torchinsky have shown in [5] that the $A_{\infty, \mathscr{R}}$ condition is strictly stronger than condition (A). As an example they show (cf. [5, p. 270]) that if the weight $w$ in $\mathbf{R}^{n}, n>1$, is $A_{\infty}$ in each variable except one where it is merely doubling, then $w$ satisfies condition (A) while $w$ does not belong to $A_{\infty, \mathscr{R}}$, as is well known.

A first result on the two weight problem was remarked by the author in [9]. We pointed out that the following generalized Fefferman-Stein's type inequality

$$
\int_{\mathbf{R}^{n}} M_{\mathscr{B}} f(y)^{p} w(y) d y \leq c \int_{\mathbf{R}^{n}}|f(y)|^{p} M_{\mathscr{B}} w(y) d y
$$

holds assuming that $\mathscr{B}$ is a Muckenhoupt basis, and that the weight $w$ satisfies condition (A). We recall that the classical Fefferman-Stein inequality for the Hardy-Littlewood maximal operator $M$ has no restriction on $w$.

At this point we mention that a particular instance of this result was previously obtained by Lin in [6]. His result is for the strong maximal operator $M_{\mathscr{R}}$ in dimension $n=2$ and with the weight $w$ satisfying the $A_{\infty, \mathscr{R}}$ condition.

The main result of this paper is

Theorem 1.3. Let $1<p<\infty$, and let $\mathscr{B}$ be a general basis satisfying $M_{\mathscr{B}}$ : $L^{s}\left(\mathbf{R}^{n}\right) \rightarrow L^{s}\left(\mathbf{R}^{n}\right)$ for all $1<s<\infty$. Suppose that $(w, v)$ is a couple of weights such that $w$ satisfies (A), and that for some $1<r<\infty$ there is a contant $c$ such that

$$
\frac{1}{|B|} \int_{B} w(y) d y\left(\frac{1}{|B|} \int_{B} v(y)^{\left(1-p^{\prime}\right) r} d y\right)^{(p-1) / r} \leq c
$$


for all $B \in \mathscr{B}$. Then

$$
M_{\mathscr{B}}: L^{p}(v) \rightarrow L^{p}(w) .
$$

It is easy to check that $\left(w, M_{\mathscr{B}} w\right)$ satisfies (8), and then we have

Corollary 1.4. Let $1<p<\infty$, and let $\mathscr{B}$ be a general basis satisfying $M_{\mathscr{B}}$ : $L^{s}\left(\mathbf{R}^{n}\right) \rightarrow L^{s}\left(\mathbf{R}^{n}\right)$ for all $1<s<\infty$. Suppose that $w$ is a weight that satisfies condition (A). Then

$$
\int_{\mathbf{R}^{n}} M_{\mathscr{B}} f(y)^{p} w(y) d y \leq c \int_{\mathbf{R}^{n}} f(y)^{p} M_{\mathscr{B}} w(y) d y .
$$

In particular, if $\mathscr{B}$ is a Muckenhoupt basis then we always have that $M_{\mathscr{B}}$ : $L^{s}\left(\mathbf{R}^{n}\right) \rightarrow L^{s}\left(\mathbf{R}^{n}\right), 1<s<\infty$, yielding (7) as a corollary.

Corollary 1.5. Let $1<p<\infty$, and let $\mathscr{B}$ be a Muckenhoupt basis. Suppose that $w$ is a weight that satisfies condition (A). Then

$$
\int_{\mathbf{R}^{n}} M_{\mathscr{B}} f(y)^{p} w(y) d y \leq c \int_{\mathbf{R}^{n}} f(y)^{p} M_{\mathscr{B}} w(y) d y .
$$

\section{PROOF OF THE THEOREM}

First we claim that $(8)$ implies

$$
M_{\mathscr{B}}: L^{p}(v) \rightarrow L^{p, \infty}(w)
$$

where $L^{p, \infty}(w)$ is the weighted Lorentz space defined by all the functions $f$ such that $\sup _{\lambda>0}\left(\lambda^{p} w\left(\left\{x \in \mathbf{R}^{n}: M_{\mathscr{B}} f(x)>\lambda\right\}\right)\right)<\infty$.

To prove (10) we just need to prove that there is a constant $c$ such that

$$
w(K) \leq \frac{c}{t^{p}} \int_{\mathbf{R}^{n}} f(y)^{p} v(y) d y,
$$

for each $t>0, f \geq 0$, and for any compact subset $K$ of $\left\{x \in \mathbf{R}^{n}: M_{\mathscr{B}}(f)(x)>\right.$ $t\}$. By the compactness of $K$ and the definition of $M_{\mathscr{B}} f$, we can find a finite collection $B_{1}, \ldots, B_{N}$ such that

$$
K \subset \bigcup_{j=1}^{N} B_{j} \quad \text { and } t<\frac{1}{\left|B_{j}\right|} \int_{B_{j}} f(y) d y
$$

for each $j=1, \ldots, N$. We now follow a well-known selecting procedure argument (cf. [2, p. 463] for instance). Let $\widetilde{B}_{1}=B_{1}$ and, once $\widetilde{B}_{1}, \ldots, \widetilde{B}_{k-1}$ have been selected, we choose $\widetilde{B}_{k}$ to be the first set in the given sequence (if any) such that

Now we claim that

$$
\left|\widetilde{B}_{k} \cap\left(\bigcup_{j=1}^{k-1} \widetilde{B}_{j}\right)\right|<\lambda\left|\widetilde{B}_{k}\right|
$$

$$
\bigcup_{j=1}^{N} B_{j} \subset\left\{x \in \mathbf{R}^{n}: M_{\mathscr{B}}\left(\chi_{\bigcup_{j=1}^{M} \widetilde{B}_{j}}\right)(x) \geq \lambda\right\} .
$$

Let $x \in \bigcup_{j=1}^{N} B_{j}$; if $x$ belongs to some $\widetilde{B}_{k}$ it is of course obvious that it is contained on the set to the right since $\lambda<1$. If, on the other hand, $x \in B_{j}$ 
for some $B_{j}$ that has been discarded in the selection process, we must have $\left|B_{j} \cap\left(\bigcup_{j=1}^{M} \widetilde{B}_{j}\right)\right| \geq \lambda\left|B_{j}\right|$, and therefore $M_{\mathscr{B}}\left(\chi_{\bigcup_{j=1}^{M} \widetilde{B}_{j}}\right)(x) \geq \lambda$. Now, since $w$ satisfies condition $(A),(12)$ yields

$$
w\left(\bigcup_{j=1}^{N} B_{j}\right) \leq c w\left(\bigcup_{j=1}^{M} \widetilde{B}_{j}\right) .
$$

This together with (11), (8), and Hölder's inequality yields the estimate

$$
\begin{aligned}
w(K) \leq & c w\left(\bigcup_{j=1}^{M} \widetilde{B}_{j}\right) \leq c \sum_{j}\left(\frac{1}{t\left|\widetilde{B}_{j}\right|} \int_{\widetilde{B}_{j}} f(y) d y\right)^{p} w\left(\widetilde{B}_{j}\right) \\
= & c \frac{1}{t^{p}} \sum_{j}\left(\frac{1}{\left|\widetilde{B}_{j}\right|} \int_{\widetilde{B}_{j}} f(y) v(y)^{1 / p} v(y)^{-1 / p} d y\right)^{p} w\left(\widetilde{B}_{j}\right) \\
\leq & c \frac{1}{t^{p}} \sum_{j}\left(\frac{1}{\left|\widetilde{B}_{j}\right|} \int_{\widetilde{B}_{j}} f(y)^{\left(p^{\prime} r\right)^{\prime}} v(y)^{\left(p^{\prime} r\right)^{\prime} / p} d y\right)^{p /\left(p^{\prime} r\right)^{\prime}} \\
& \times\left(\frac{1}{\left|\widetilde{B}_{j}\right|} \int_{\widetilde{B}_{j}} v(y)^{-p^{\prime} r / p} d y\right)^{p / p^{\prime} r} \frac{w\left(\widetilde{B}_{j}\right)}{\left|\widetilde{B}_{j}\right|}\left|\widetilde{B}_{j}\right| \\
\leq & c K \frac{1}{t^{p}} \sum_{j}\left(\frac{1}{\left|\widetilde{B}_{j}\right|} \int_{\widetilde{B}_{j}} f(y)^{\left(p^{\prime} r\right)^{\prime}} v(y)^{\left(p^{\prime} r\right)^{\prime} / p} d y\right)^{p /\left(p^{\prime} r\right)^{\prime}}\left|\widetilde{B}_{j}\right| .
\end{aligned}
$$

Denote $E_{j}=\widetilde{B}_{j} \backslash \bigcup_{i=1}^{j-1} \widetilde{B}_{i}$, so that $\left\{E_{j}\right\}$ is a disjoint family with $E_{j} \subset \widetilde{B}_{j}$ and $\left|\widetilde{B}_{j}\right|<\frac{1}{1-\lambda}\left|E_{j}\right|$. Then

$$
\begin{aligned}
w(K) & \leq c \frac{1}{t^{p}} \sum_{j}\left(\frac{1}{\left|\widetilde{B}_{j}\right|} \int_{\widetilde{B}_{j}} f(y)^{\left(p^{\prime} r\right)^{\prime}} v(y)^{\left(p^{\prime} r\right)^{\prime} / p} d y\right)^{p /\left(p^{\prime} r\right)^{\prime}}\left|E_{j}\right| \\
& \leq \frac{c}{t^{p}} \sum_{j} \int_{E_{j}} M_{\mathscr{B}}\left(f^{\left(p^{\prime} r\right)^{\prime}} v^{\left(p^{\prime} r\right)^{\prime} / p}\right)(y)^{p /\left(p^{\prime} r\right)^{\prime}} d y \\
& \leq \frac{c}{t^{p}} \int_{\mathbf{R}^{n}} M_{\mathscr{B}}\left(f^{\left(p^{\prime} r\right)^{\prime}} v^{\left(p^{\prime} r\right)^{\prime} / p}\right)(y)^{p /\left(p^{\prime} r\right)^{\prime}} d y \\
& \leq \frac{c}{t^{p}} \int_{\mathbf{R}^{n}} f(y)^{p} v(y) d y,
\end{aligned}
$$

since, by hypothesis, $M_{\mathscr{B}}: L^{s}\left(\mathbf{R}^{n}\right) \rightarrow L^{s}\left(\mathbf{R}^{n}\right), 1<s<\infty$.

To conclude the proof of the theorem we observe first that we always have

$$
M_{\mathscr{B}}: L^{\infty}(v) \rightarrow L^{\infty}(w)
$$

Now, denoting condition (8) by $A_{p, r}$, we see that $(w, v)$ satisfies $A_{\bar{p}, \bar{r}}$ for some $1<\bar{p}<p, 1<\bar{r}<\infty$; in fact $(p-1) / r+1<\bar{p}<p, 1<\bar{r}<$ 
$(\bar{p}-1) r /(p-1)$ will do it:

$$
\begin{aligned}
& \frac{1}{|B|} \int_{B} w(y) d y\left(\frac{1}{|B|} \int_{B}\left(v(y)^{-1}\right)^{\bar{r} /(\bar{p}-1)} d y\right)^{(\bar{r} /(\bar{p}-1))^{-1}} \\
& \quad \leq \frac{1}{|B|} \int_{B} w(y) d y\left(\frac{1}{|B|} \int_{B}\left(v(y)^{-1}\right)^{r /(p-1)} d y\right)^{(r /(p-1))^{-1}} \leq K .
\end{aligned}
$$

By the above argument $A_{\bar{p}, \bar{r}}$ and $M_{\mathscr{B}}: L^{s}\left(\mathbf{R}^{n}\right) \rightarrow L^{s}\left(\mathbf{R}^{n}\right), 1<s<\infty$, yield

$$
M_{\mathscr{B}}: L^{\bar{p}}(v) \rightarrow L^{\bar{p}, \infty}(w) .
$$

This together with (13) implies $M_{\mathscr{B}}: L^{p}(v) \rightarrow L^{p}(w)$ by the Marcinkiewicz interpolation theorem. This concludes the proof of the theorem.

\section{REFERENCES}

1. A. Córdoba, On the Vitali covering properties of a differentiation basis, Studia Math. $\mathbf{5 7}$ (1976), 91-95.

2. J. Garcia-Cuerva and J. L. Rubio de Francia, Weighted norm inequalities and related topics, North-Holland Math. Stud., vol. 116, North-Holland, Amsterdam, 1985.

3. R. A. Hunt, D. S. Kurtz, and C. J. Neugebauer, A note on the equivalence of $A_{p}$ and Sawyer's condition for equal weights, Conf. Harmonic Analysis in honor of A. Zygmund (W. Beckner, A. P. Calderón, R. Fefferman, and P. W. Jones, ed.), Wadsworth, Belmont, CA, 1981, pp. 156-158.

4. B. Jawerth, Weighted inequalities for maximal operators: linearization, localization, and factorization, Amer. J. Math. 108 (1986), 361-414.

5. B. Jawerth and A. Torchinsky, The strong maximal function with respect to measures, Studia Math. 80 (1984), 261-285.

6. K. C. Lin, Harmonic analysis on the bidisc, Thesis, U.C.L.A., 1984.

7. B. Muckenhoupt, Weighted norm inequalities for the Hardy-Littlewood maximal function, Trans. Amer. Math. Soc. 165 (1972), 207-226.

8. C. J. Neugebauer, Inserting $A_{p}$-weights, Proc. Amer. Math. Soc. 87 (1983), 644-648.

9. C. Pérez, Weighted norm inequalities for general maximal operators, Proceedings of a Conference in Harmonic Analysis and Partial Differential Equations in honor of J. L. Rubio de Francia (J. Bruna and F. Soria, eds.), Publ. Mat. 34 (1990).

10. $\ldots$, On sufficient conditions for the boundedness of the Hardy-Littlewood maximal operator between weighted $L^{p}$-spaces with different weights, preprint, 1990.

11. E. T. Sawyer, A characterization of a two weight norm weight inequality for maximal operators, Studia Math. 75 (1982), 1-11.

12. __ Two weight norm inequalities for certain maximal and integral operators, Lectures Notes in Math., vol. 908, Springer-Verlag, New York, 1982, pp. 102-127.

Department of Mathematical Sciences, New Mexico State University, Las Cruces, New Mexico 88003

Current address: Department of Mathematics, Universidad Autonoma, Madrid 28049, Spain 